\title{
APLICABILIDADE DO ÍNDICE ADIPOSIDADE CORPORAL NA ESTIMATIVA DO PERCENTUAL DE GORDURA DE JOVENS MULHERES BRASILEIRAS
}

\author{
APPLICABILITY OF BODY ADIPOSITY INDEX IN ESTIMATING BODY FAT \\ OF YOUNG BRAZILIAN WOMEN
}

\author{
APLICABILIDAD DEL ÍNDICE DE ADIPOSIDAD CORPORAL EN LA ESTIMATIVA DEL PORCENTUAL \\ DE GRASA DE JÓVENES MUJERES BRASILEÑAS
}

\author{
Jennifer Dias (Educadora Física) ${ }^{1}$ \\ Márcia de Ávila (Educadora Física) \\ Vinicius de Oliveira Damasceno \\ (Educador Físico) $^{2}$ \\ Reginaldo Gonçalves \\ (Educador Físico) $^{3}$ \\ Fernando Policarpo Barbosa \\ (Educador Físico) $^{4}$ \\ Joel Alves Lamounier \\ (Médico Pediatra) ${ }^{5}$ \\ Jeferson Macedo Vianna \\ (Educador Físico) $^{6}$ \\ 1. Universidade Federal de Viçosa, \\ Viçosa, MG, Brasil. \\ 2. Universidade Federal de \\ Pernambuco, Campus Recife, \\ PE, Brasil. \\ 3. Universidade Federal de Minas \\ Gerais, Campus Pampulha, Belo \\ Horizonte, MG, Brasil. \\ 4. Faculdades Integradas de Patos, \\ Patos, PB, Brasil. \\ 5. Universidade Federal de São \\ João del Rei, São João del Rei, \\ MG, Brasil. \\ 6. Universidade Federal de Juiz de \\ Fora, Juiz de Fora, MG, Brasil.
}

\section{Correspondência:}

Rua Aviador Severiano Lins, 466 apt 1303 - Boa Viagem, Recife, PE.

51020-060 - Brasil.

vinicius.damasceno.gmail.com

vinicius.damasceno@ufpe.br

\begin{abstract}
RESUMO
Introdução: A estimativa da composição corporal é um campo de estudo importante no prognóstico e diagnóstico de enfermidades degenerativas. Objetivo: Analisar a aplicabilidade do Índice de adiposidade corporal (IAC) e o método de dobras cutâneas. Métodos: A amostra foi constituída por 19 adultas jovens com média de idade 24,53 \pm 2,65 anos, submetidas à avaliação antropométrica (circunferências e dobras cutâneas) e absorsiometria por dupla emissão de raio X (DXA). Os valores estimados foram comparados ao valor de referência por meio do teste $t$ pareado e pela análise do nível de associação entre os métodos pela correlação de Pearson; o nível de significância foi $p<0,05$. Resultados: Não apontaram diferenças significativas entre o percentual de gordura estimado pelo IAC e os valores de referência, porém, tiveram nível de associação moderado $(r=0,627)$. No entanto, o método de dobras cutâneas apresentou diferença estatística significante $(p>0,05)$ com nível de associação forte $(r=0,879)$. Conclusão: Embora o IAC não apresente diferença para os valores estimados, fica evidente a necessidade de mais estudos sobre a aplicabilidade do método na população brasileira.
\end{abstract}

Palavras-chave: composição corporal, mulheres, pregas cutâneas.

\section{ABSTRACT}

Introduction: The estimation of body composition is an important study field in the prognosis and diagnosis of degenerative diseases. Objective: To analyze the applicability of the Body Adiposity Index (BAl) and the skinfold thickness method. Methods: The sample consisted of 19 young adult women with a mean age $24.53 \pm 2.65$ years, who underwent anthropometric measurements (circumferences and skinfold thickness) and dual energy X-ray absorptiometry (DEXA). The estimated values were compared to the reference value by using the paired $t$ test and by analysis of the level of association between the methods through the Pearson correlation. The level of significance was $p<0.05$. Results: Showed no significant differences between the fat percentage estimated by the BAl and the reference values, however had moderate level of association $(r=0.627)$. Nevertheless, the skinfold thickness method showed statistically significant difference ( $p>0.05)$ with a strong association level $(r=0.879)$. Conclusion: Although the BAl does not show difference to the estimated values, it is clear that further studies on the applicability of the method in the Brazilian population are necessary.

Keywords: body composition, women, skinfolds thickness.

\section{RESUMEN}

Introducción: La estimativa de la composición corporal es un campo de estudio importante en el pronóstico y diagnóstico de enfermedades degenerativas. Objetivo: Analizar la aplicabilidad del Índice de Adiposidad Corporal (IAC) y el método de pliegues cutáneos. Métodos: La muestra fue constituida por 19 adultas jóvenes con promedio de edad de 24,53 $\pm 2,65$ años, sometidas a evaluación antropométrica (circunferencias y pliegues cutáneos) y absorciometría por doble emisión de rayo X (DXA). Los valores estimados fueron comparados al valor de referencia por medio del test t pareado y por el análisis del nivel de asociación entre los métodos por la correlación de Pearson; el nivel de significancia fue de $p<0,05$. Resultados: No apuntaron diferencias significativas entre el porcentual de grasa estimado por el IACy los valores de referencia, no obstante, tuvieron nivel de asociación moderado $(r=0,627)$. Por otro lado, el método de pliegues cutáneos presentó diferencia estadística significativa $(p>0,05)$ con nivel de asociación fuerte $(r=0,879)$. Conclusión: Aunque el IAC no presente diferencia para los valores estimados, queda evidente la necesidad de más estudios sobre la aplicabilidad del método en la población brasileña.

Palabras clave: composición corporal, mujeres, pliegues cutáneos. 


\section{INTRODUÇÃO}

A determinação dos componentes da composição corporal permite estabelecer prognósticos de ocorrência de enfermidades degenerativas, assim como, o diagnóstico de intervenção, tornando-se um procedimento de extrema relevância em diferentes áreas da saúde ${ }^{1,2}$. Para tanto, há uma gama de equipamentos e métodos que permitem determinar o gordura (\%G), da massa corporal magra (MCM) e da massa de gordura (MG) $)^{3,4}$.

No entanto, diversas discussões que tange as investigações no campo da antropometria implica no questionamento sobre o custo e aplicabilidade dos métodos e equipamentos na estimativa da composição corporal em diferentes populações ${ }^{5-9}$. É fato, que uma equação de estimativa da composição corporal oriunda de população, necessita ser analisada e validada antes de ser aplicada em outra população ${ }^{5,7}$. A importância de se analisar a aplicabilidade de equações de estimativa da composição corporal na população brasileira, centra-se na utilização de método de baixo custo como a estimativa do \%G, pelo índice de massa corporal (IMC), a perimetria e as dobras cutânea, que permitem diagnosticar os aspectos nutricionais ${ }^{10,11}$, como também, fomentar parâmetros para estabelecer as cargas de trabalho nos programas de exercícios como forma de prevenção de doenças degenerativas não transmissíveis ${ }^{2,12}$.

Dentre os métodos de baixo custo aplicados, o IMC é o que tem sofrido mais críticas nos últimos anos, por não analisar os componentes da composição corporal. Da mesma forma, o método de dobras cutâneas também vem recebendo uma série de críticas quanto a sua aplicabilidade e confiabilidade ${ }^{4,9}$. Com base nessas críticas, Bergman, et al. ${ }^{12}$ propuseram um método que permitisse a estimativa do \%G tanto para homens como para mulheres por meio de medidas da circunferência do quadril e da estatura, denominado por índice de adiposidade corporal - IAC = ((circunferência do quadril) / ((altura) $(1,5))$-18)). Em seu estudo, foi observado um $R^{2}=0,959$ para mulheres e de 0,957 para homens. Entretanto, o IAC ainda não foi aplicado e testado na população brasileira. Como base nessas observações, o presente estudo tem por objetivo verificar a aplicabilidade do IAC em jovens mulheres brasileiras em comparação ao método de dobras cutâneas tendo como referência da composição corporal os valores obtidos pela absorsiometria por dupla emissão de raios-X (DXA).

\section{MATERIAIS E MÉTODO}

Na presente investigação de caráter correlacional de corte transversal, onde se busco estabelecer o nível de associação entre métodos de estimativa da composição corporal de jovens mulheres brasileiras. Para tanto, 19 voluntárias com idade entre 20 e 30 anos, residentes na cidade de Viçosa, MG, Brasil, foram convidadas a participar do estudo. Como critérios de inclusão as voluntárias deveriam ter Índice de massa corporal (IMC $<30 \mathrm{~kg} / \mathrm{m}^{2}$ ) e, não estar menstruadas no dia do teste. As voluntárias que concordaram em participar do estudo, foram informadas e esclarecidas dos riscos do estudo e convidadas a assinar o termo de consentimento livre e esclarecido em conforme normas éticas exigidas pela Resolução no 196 de 10 de outubro de 1996 (Conselho Nacional de Saúde). O projeto de pesquisa foi submetido e aprovado pelo Comitê de Ética da Universidade Federal de Viçosa, MG, Brasil (Protocolo 028/2011).

A coleta de dados foi realizada no período de dezembro de 2011 a fevereiro de 2012. As voluntárias permaneceram com indumentária apropriada para a realização dos testes, usando short, top e descalças. A determinação da massa corporal foi realizada utilizando-se uma balança portátil da marca Filizola (Brasil), com precisão de 100g; para a medida da estatura utilizou-se do estadiômentro portátil da marca Sanny, com escala em milímetros. A circunferência de cintura foi realizada no ponto médio entre a última costela e a crista ilíaca com o braço flexionado a $90^{\circ}$, e a medida de circunferência do quadril foi realizada no ponto de maior perímetro glúteo ${ }^{5}$, para tanto, utilizou-se da fita antropométrica metálica de 2 metros, da marca Sanny.

A partir dos valores de estatura e circunferência do quadril, foi calculado o IAC através da equação: IAC $(\%)=C Q(\mathrm{~cm}) /$ altura $\mathrm{x}$ raiz quadrada da altura $(\mathrm{m})-18^{12}$. Foi calculado também o IMC utilizando massa corporal e estatura por meio da seguinte razão: massa corporal $(\mathrm{kg})$ /estatura $\left(\mathrm{m}^{2}\right)$. E a densidade corporal (DC) foi transformada em $\%$ G através da fórmula de $\operatorname{Siri}^{13}$.

Para a estimativa da densidade corporal aplicou-se o método de dobras cutâneas (DC). Foram utilizadas as equações de sete e três dobras cutâneas de Jackson, Pollock e Ward ${ }^{14}$ para mulheres com idades entre 18 e 55 anos. As equações foram eleitas por já terem estudo de validação para a população brasileira ${ }^{8,15}$. As DC foram mensuradas no hemicorpo direito com o indivíduo em posição ortostática com a musculatura relaxada, as DC mensuradas para o protocolo de sete dobras foram: subescapular, axilar média, tríceps, coxa, supra-ilíaca, abdômen e peitoral e para o protocolo de três dobras: tríceps, supra-ilíaca e coxa ${ }^{8}$. As medidas das dobras cutâneas foram realizadas pelo plicômetro da marca Sanny, com acuidade de 0,5 mm e variação de 0 a $55 \mathrm{~mm}$. Para a estimativa do percentual de gordura (\%G) foi utilizada a equação de $\mathrm{Siri}^{16}$ a partir do resultado de densidade corporal.

O método utilizado como padrão-ouro na determinação da composição corporal das mulheres foi a absorsiometria por dupla emissão de raio $X(D X A)$. A DXA, um procedimento de imageamento de alta tecnologia que permite a quantificação da gordura e do músculo, assim como o conteúdo mineral ósseo ${ }^{17}$. O equipamento utilizado foi o densitômetro, marca GE Healthcare (Lunar Prodigy Advance DXA System versão 13.31), manuseado por um técnico capacitado. Todos os exames foram feitos na Divisão de Raio X e Densitometria Óssea da Divisão de Saúde da Universidade Federal de Viçosa. O exame foi realizado com o indivíduo deitado em decúbito dorsal sobre uma mesa, onde a fonte e o detector foram passados através do corpo com uma velocidade lenta.

\section{Análise Estatística}

Os dados foram tratados em um primeiro momento observando-se a curva de distribuição por meio dos testes Shapiro-Wilk e o gráfico Q-Q de normalidade, sendo observada curva normal para todas as variáveis do estudo. Para a comparação entre os valores estivados pelos métodos de DC e IAC com os determinados pela DXA foi aplicado o teste t pareado. A determinação do nível de correlação entre os métodos indiretos e o método direto foi aplicado o coeficiente de correlação linear R de Pearson e Alpha de Conbrach. Para análise dos escores residuais aplicou-se o teste de Bland \& Altman. Para aceitação das diferenças entre os valores estimados da composição corporal e o valor de referencia, assim como para o nível de correlação entre os métodos foi adotado um nível de significância $p<0.05$. Os resultados serão apresentados por meio da estatística de tendência central (média) e de dispersão (desvio padrão - Dp).

\section{RESULTADOS}

As características amostrais de idade, massa corporal, estatura e perimetria estão descritos na tabela 1. A média de idade das voluntárias encontra-se no intervalo determinado para cada protocolo.

Os resultados obtidos na análise dos dados indicam que os métodos de estimativa da composição corporal pela DC foram significativamente diferentes do valor de referência $(p>0,05)$. Isso demonstra menor precisão método de DC na estimativa do \%G de jovens mulheres brasileiras. No entanto, o índice de adiposidade corporal (IAC) não apresentou diferença estatística significativa $(p=0,01)$ tabela 2 .

A análise do coeficiente de correlação linear $R$ de Pearson e aná- 
Tabela 1. Características de jovens mulheres brasileiras submetidas à avaliação antropométrica $(\mathrm{n}=19)$.

\begin{tabular}{c|c|c|c}
\hline & Min. & Max. & Media \pm Dp \\
\hline Idade $($ anos $)$ & 20 & 30 & $24,53 \pm 2,65$ \\
\hline Massa corporal $(\mathrm{kg})$ & 44,0 & 75,0 & $57,62 \pm 7,50$ \\
\hline Estatura $(\mathrm{m})$ & 1,49 & 1,73 & $1,63 \pm 0,06$ \\
\hline IMC $\left(\mathrm{kg} / \mathrm{m}^{2}\right)$ & 19,3 & 28,4 & $21,80 \pm 2,53$ \\
\hline Cintura $(\mathrm{cm})$ & 60 & 79 & $68,74 \pm 5,14$ \\
\hline Quadril $(\mathrm{cm})$ & 90 & 108 & $97,63 \pm 5,01$ \\
\hline
\end{tabular}

Tabela 2. Valores para o percentual de gordura de jovens mulheres brasileiras $(n=19)$ estimados por diferentes métodos de estimativa.

\begin{tabular}{c|c|c|c}
\hline & Mín. & Máx. & Média \pm Dp \\
\hline DEXA & 17 & 38 & $28,68 \pm 5,55$ \\
\hline IAC & 24,0 & 34,5 & $29,11 \pm 3,00$ \\
\hline Equação 7 DC. & 16,0 & 32,2 & $24,19 \pm 4,46^{* *}$ \\
\hline Equação 3 DC. & 17,26 & 31,30 & $24,69 \pm 4,05^{* *}$ \\
\hline
\end{tabular}

$* *=p<0,01 ; \mathrm{DEXA}=$ absorsiometria por dupla emissão de raio $X ; \mid \mathrm{AC}=$ Índice de adiposidade corporal, $D C=$ Dobras cutáneas

lise do Alpha de Cronbach indicaram valores significativos entre o \%G estimado pelo IAC e o determinado pelo DXA $(r=0,627 ; p=0,01)$. No entanto, só foi observada uma associação de 39,3\% entre os métodos pelo Alpha de Cronbach. As equações de sete e de três dobras cutâneas apresentaram correlação significativas com o DXA ( $r=0,879$; $p=0,01)$ e $(r=0,887 ; p=0,01)$ respectivamente, e uma associação de $77,3 \%$ para a equação de sete dobras e de 78,7\% para a equação de três dobras cutâneas. Os resultados obtidos para a correlação indica melhores índices de associação entre o método de dobras cutâneas com o padrão-ouro.

A análise dos escores residuais pelo método de Bland \& Altman (figura 1) demonstra que o método do IAC tanto pode subestimar como superestimar o \% $\mathrm{G}$ das voluntárias.

Nas figuras 2 e 3, podem ser analisados o comportamento dos escores residuais para as equações de sete e três DC. Na equação de sete DC observa-se uma tendência em subestimar a maior parte das voluntárias, o que reduz a sua validação para a população brasileira. O mesmo comportamento pode ser observado para a equação de três DC, porém essa equação subestimou aproximadamente $68 \%$ da amostra estuda. Esse resultado contraindica a aplicação da equação de três DC na estimativa do \%G de jovens mulheres brasileiras. Para a análise dos escores residuais foi adotado um erro de $\pm 5 \%$ entre os métodos de estimativa e o de referência.

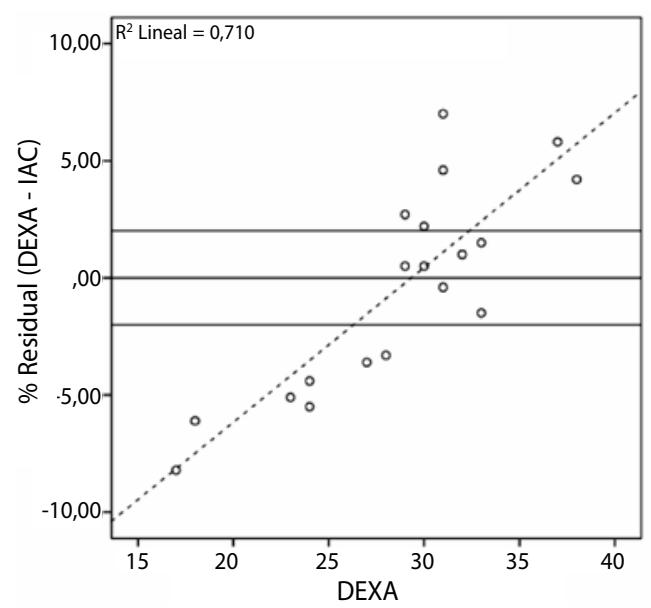

Figura 1. Distribuição dos escores residuais para a DEXA e o IAC de 19 jovens muIheres brasileiras.

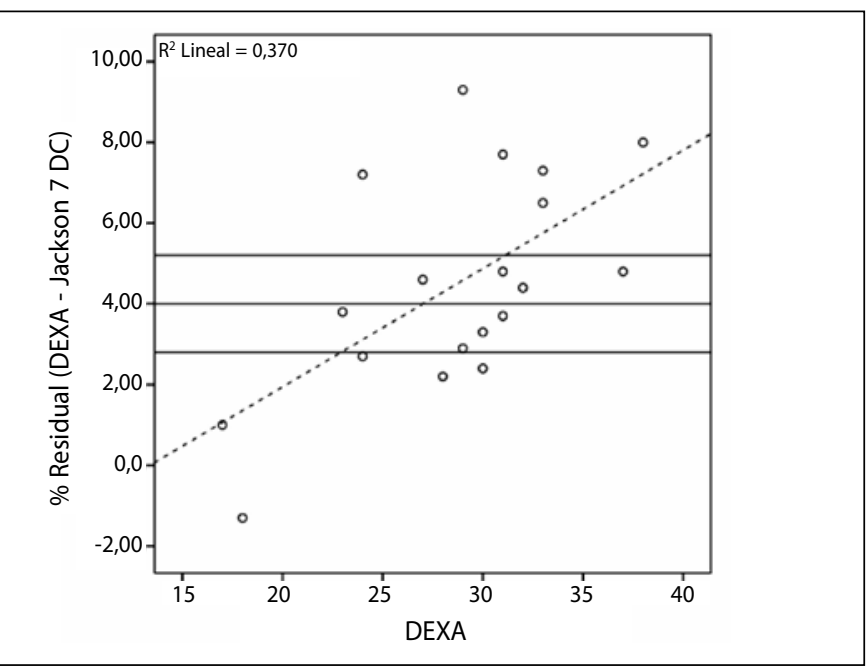

Figura 2. Distribuição dos escores residuais para a DEXA e a equação de sete dobras cutâneas para jovens mulheres brasileiras.

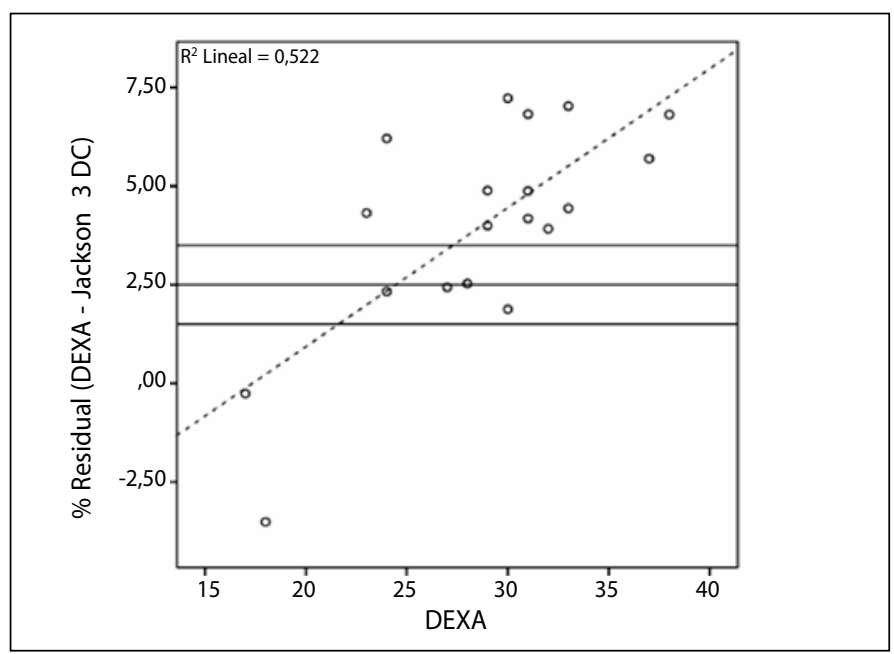

Figura 3. Distribuição dos escores residuais para a DEXA e a equação de três dobras cutâneas para jovens mulheres brasileiras.

\section{DISCUSSÃO}

A relevância do estudo está em analisar a eficácia do método na estimativa da gordura corporal na população brasileira, uma vez que os aspectos socioculturais podem ter influência direta na morfologia da população 9,18. Além disso, a determinação da eficácia do IAC permite diagnósticos acurados da composição corporal, já que, o índice de massa corporal (IMC) apresenta divergências na determinação do sobrepeso e da obesidade na população brasileira"1. A utilização de um método validado para a estimativa da composição corporal na população brasileira em conjunto como outras medidas clínicas, poderia permitir o estabelecimento de estratégias de Saúde Pública com maior eficiência na prevenção primária ou secundária de enfermidades, principalmente no que concernem as doenças crônicas degenerativas não transmissíveis ${ }^{19,21}$.

$\mathrm{Na}$ análise do IAC, o método apresentou resultados satisfatórios quando comparado aos valores de referência, não apresentaram diferenças estatísticas significativas $(p>0,05)$ na estimativa do \%G. Porém, o IAC não apresentou acurácia na estimativa dos valores superiores do \%G da amostra, assim como, para os valores inferiores do \%G, indicando tendência em super ou subestimar em média 36\% o \%G da amostra. O que seria consistente com os dados de Mohammadreza, et al.' que analisaram a capacidade preditiva do IAC, apontaram uma baixa acurácia do método, o que seria convergente com os resultado obtidos no presente estudo. Os resultados de Mohammadreza et al.' confirmam os 
obtidos por Santos Silva, Petroski e Peres ${ }^{22}$ que analisaram dois métodos o IMC e a circunferência da cintura (CC) na estimativa do \%G, os quais, se mostraram eficazes na predição de quadros hipertensivos.

No que concerne a dispersão dos dados, Bergman, et al.' ao estabelecer a reta de regressão como 1733 indivíduos de ambos os sexos de descendência mexicana obteve uma baixa dispersão. Comportamento similar também observado pelos autores quando aplicaram o IAC em uma população de afros-americanos, apontando uma boa aplicabilidade do método. No entanto, no presente estudo houve uma grande dispersão dos dados, o que pode ser explicado pelo tamanho amostral de 19 mulheres. Outro ponto que poderia explicar os resultados obtidos por Bergman et al. ${ }^{12}$ seria o nível de associação entre a DXA e medidas antropométricas com o objetivo de estimar a gordura visceral de homens e mulheres. Porém, os níveis de associação apresentam uma variação de fraca a moderada ${ }^{23,24}$. O que é convergente para os resultados observados para a correlação no presente estudo, embora tenha sido significativa indicando uma relação entre a DXA e o IAC, a mesma foi moderada ( $r=0,5$ a 0,6), o que indica uma convergência entre os estudos ${ }^{25}$. Porém os resultados para o Alpha de Cronbach demonstra uma baixa acuidade (39,3\%) para o IAC indicando que o método necessita ser melhor analisado antes de ser utilizado na população brasileira, em contrapartida a utilização das equações de estimativa que tem como parâmetros as dobras cutâneas 8,14,15.

A afirmativa acima perde um pouco da sua plausibilidade devido aos resultados obtidos para a estimativa do \%G por meio das equações de três dobras cutâneas de Jackson, Pollock, Ward ${ }^{14}$, a qual, tendeu a superestimar a composição corporal de $73 \%$ da amostra, o que indica uma baixa acuidade da equação, o que é divergente aos resultados obtidos em estudo anteriores ${ }^{8,15}$. A mesma tendência de baixa precisão foi observada para a equação de sete dobras cutâneas, subestimando $42 \%$ da amostra, o que poderia explicar a discordância entre os resultados do presente estudo e de estudos anteriores, possivelmente, seria a utilização da equação para densidade corporal para hispânicos ${ }^{8}$.

\section{REFERÊNCIAS}

1. Mohammadreza B, Farzad H, Davoud K, Fereidoun Prof AF. Prognostic significance of the complex "Visceral Adiposity Index" vs. simple anthropometric measures: Tehran lipid and glucose study. Cardiovascular diabetology. 2012;11:20. Epub 2012/03/08.

2. Jacobs N, De Bourdeaudhuij I, Thijs H, Dendale P, Claes N. Effect of a cardiovascular prevention program on health behavior and BMI in highly educated adults: a randomized controlled trial. Patient education and counseling. 2011;85(1):122-6.

3. Jaffrin MY, Morel H. Body fluid volumes measurements by impedance: A review of bioimpedance spectroscopy (BIS) and bioimpedance analysis (BIA) methods. Medical engineering \& physics. 2008;30(10):1257-69. Epub 2008/08/05.

4. Casey AF. Measuring body composition in individuals with intellectual disability: a scoping review. Journal of obesity. 2013;2013:628428. Epub 2013/06/14

5. Heyward VH. Advanced fitness assessment and exercise prescription. 6th ed. Champaign, IL: Human Kinetics; 2010. xiii, 465 p.p.

6. Lustgarten MS, Fielding RA. Assessment of analytical methods used to measure changes in body composition in the elderly and recommendations for their use in phase II clinical trials. The journal of nutrition, health \& aging. 2011;15(5):368-75. Epub 2011/04/30.

7. Pollock ML, Jackson AS. Research progress in validation of clinical methods of assessing body composition. Medicine and science in sports and exercise. 1984;16(6):606-15. Epub 1984/12/01.

8. Bottaro MF, Heyward VH, Bezerra RFA, Wagner DR. Skinfold method vs dual-energy $x$-ray absorptiometry to assess body composition in normal and obese women. J Exerc Physiol Online. 2002;5(2):11-8.

9. Rezende FAC, Rosado LEFPL, Priore SE, Franceschini SdCC. Aplicabilidade de equações na avaliação da composição corporal da população brasileira; Applicability of equations in assessing the body composition of the Brazilian population. Rev nutr. 2006;19(3):357-67.

10. Cunha DB, de Almeida RM, Sichieri R, Pereira RA. Association of dietary patterns with BMI and waist circumference in a low-income neighbourhood in Brazil. The British journal of nutrition. 2010;104(6):908-13.

11. Anjos LA, Teixeira Fda C, Wahrlich V, Vasconcellos MT, Going SB. Body fat percentage and body mass index in a probability sample of an adult urban population in Brazil. Cadernos de saude publica. 2013;29(1):73-81. Epub 2013/02/02.

12. Bergman RN, Stefanovski D, Buchanan TA, Sumner AE, Reynolds JC, Sebring NG, et al. A better index of body adiposity. Obesity. 2011;19(5):1083-9.

13. Siri WE. Body composition from fluid spaces and density: analysis of methods. 1961. Nutrition. 1993;9(5):480-91; discussion,92.

14. Jackson AS, Pollock ML, Ward A. Generalized equations for predicting body density of women. Medicine and science in sports and exercise. 1980;12(3):175-81. Epub 1980/01/01.
Muito embora, Petroski e Pire-Neto tenha utilizado a equação para o cálculo da densidade corporal para caucasianas ${ }^{14,15}$. Devido a essa divergência pode-se apontar a inexperiência dos avaliadores no momento da obtenção das dobras ${ }^{9,18,26}$ a qual afetaria diretamente os resultados. Esse pontos podem ser considerados como limitações do presente estudo, uma vez que foi utilizada o calculo para a densidade corporal de caucasianos, assim como em estudo anterior ${ }^{15}$, mais ressalta-se que não foi observado erro intra-avaliador como recomendado ${ }^{18}$. Quanto às equações cabe ressaltar que o Alpha de Cronbach foi superior ao calculado para o IAC, sendo que, a equação de três dobras apresentou uma concordância de $78,7 \%$ o que aponta um bom nível de acuidade $^{27}$, no entanto, a equação foi a que apresentou maio tendência em subestimar a amostra, comportamento ratificado pela analise dos escores residuais de Band \& Altman ${ }^{28}$.

Os resultados do presente estudo indicaram a necessidade de ampliar as investigações no que tange a validade do IAC na população brasileira, para tanto, recomenda que estudos complementares sejam realizados em uma amostra maior que englobe indivíduos de ambos os sexos e em diferentes faixas etárias.

\section{CONCLUSÃO}

Os resultados obtidos permite concluir que os métodos aplicados para a estimativa da composição corporal de jovens mulheres brasileiras não apresentaram a acuidade necessária para que possam ser aplicadas. Embora o IAC não tenha apresentado diferenças significativas ( $p>0,05)$, quando comparado aos valores de referência, porém, observa-se um nível de associação moderados $(r=0,627)$ entre o IAC e o DXA. Fica evidenciado a necessidade de estudo complementares com uma amostra significativa da população brasileira.

Todos os autores declararam não haver qualquer potencial conflito de interesses referente a este artigo.

15. Petroski EL, Pires Neto CS. Validação de equações antropométricas para a estimativa da densidade corporal em mulheres. Revista Brasileira de Atividade Física \& Saúde. 2012;1 (2):65-73.

16. Siri WE. The gross composition of the body. Advances in biological and medical physics. 1956;4:239-80.

17. Toombs RJ, Ducher G, Shepherd JA, De Souza MJ. The impact of recent technological advances on the trueness and precision of DXA to assess body composition. Obesity. 2012;20(1):30-9. Epub 2011/07/16.

18. Guedes DP. Procedimentos clínicos utilizados para análise da composição corporal; Clinical procedures used for analysis of the body composition. Rev bras cineantropom desempenho hum. 2013;15(1):113-29.

19. Deibert P, Konig D, Vitolins MZ, Landmann U, Frey I, Zahradnik HP, et al. Effect of a weight loss intervention on anthropometric measures and metabolic risk factors in pre- versus postmenopausal women. Nutrition journal. 2007;6:31. Epub 2007/10/27.

20. Momesso DP, Bussade I, Lima GAB, Fonseca LPC, Russo LAT, Kupfer R. Body composition, metabolic syndrome and insulin resistance in type 1 diabetes mellitus. Arquivos Brasileiros de Endocrinologia \& Metabologia. 2011;55(3):189-93.

21. Silva DA, Petroski EL, Peres MA. Accuracy and measures of association of anthropometric indexes of obesity to identify the presence of hypertension in adults: a population-based study in Southern Brazil. European journal of nutrition. 2013;52(1):237-46. Epub 2012/02/04.

22. Santos Silva DA, Petroski EL, Peres MA. Is high body fat estimated by body mass index and waist circumference a predictor of hypertension in adults? A population-based study. Nutrition journal. 2012;11:112. Epub 2012/12/19.

23. Vatanparast H, Chilibeck PD, Cornish SM, Little JP, Paus-Jenssen LS, Case AM, et al. DXA-derived abdominal fat mass, waist circumference, and blood lipids in postmenopausal women. Obesity. 2009;17(8):1635-40. Epub 2009/04/04.

24. Elisha B, Messier V, Karelis A, Coderre L, Bernard S, Prud'homme D, et al. The Visceral Adiposity Index: Relationship with cardiometabolic risk factors in obese and overweight postmenopausal women - A MONET group study. Applied physiology, nutrition, and metabolism = Physiologie appliquee, nutrition et metabolisme. 2013;38(8):892-9. Epub 2013/07/17.

25. Bewick V, Cheek L, Ball J. Statistics review 7: Correlation and regression. Crit Care. 2003;7(6):451-9. Epub 2003/11/20.

26. Dwyer GB, Davis SE, American College of Sports Medicine. ACSM's health-related physical fitness assessment manual. Philadelphia: Lippincott Williams \& Wilkins; 2005. xii, 180 p. p.

27. Cronbach LJ, Shavelson RJ. My current thoughts on coefficient alpha and successor procedures Educational and Psychological Measurement. 2004;64(3):391-418.

28. Hopkins WG. Bias in Bland-Altman but not regression validity analyses. Sportscience. 2004;8(4). 